\title{
Assessment of an Immersion Technique for Generating of Borrelia burgdorferi-Infected and Infectious Ixodes scapularis and Ixodes ricinus Ticks
}

\section{Djamel Tahir ${ }^{1 *}$, Alec Evans ${ }^{1}$, Nouha Lekouch' ${ }^{1}$, Frans Jongejan², Valérie Choumet ${ }^{3}$, Byron Blagburn ${ }^{4}$, Reinhard K Straubinger ${ }^{5}$ and Marie Varloud ${ }^{6}$}

${ }^{1}$ Clinvet Morocco, Mohammedia, Morocco

${ }^{2}$ Vectors and Vector-borne Diseases Research Programme, Department of Veterinary Tropical Diseases, Faculty of Veterinary Science, University of Pretoria, Onderstepoort, South Africa

${ }^{3}$ Environnement et Risques Infectieux, Institut Pasteur, Paris, France

${ }^{4}$ College of Veterinary Medicine, Auburn University, Auburn, Alabama, USA

${ }^{5}$ Institute for Infectious Diseases and Zoonoses, Bacteriology and Mycology, Faculty

of Veterinary Medicine, Ludwig-Maximilians-University Munich, Munich, Germany

${ }^{6}$ Ceva Santé Animale, Libourne, France

*Corresponding Author: Djamel Tahir, Clinvet Morocco, Mohammedia,

Morocco.
Received: July 11, 2020

Published: August 31, 2020

(C) All rights are reserved by Djamel Tahir., et al.

\begin{abstract}
Experimental infection of ticks with pathogens such as spirochetes of the genus Borrelia (B.), is a critical step to better understand the mechanisms and the kinetics of infection. At present, four procedures for infection of ticks with B. burgdorferi have been described: (i) feeding ticks on infected rodents; (ii) tick immersion in a solution containing the spirochetes; (iii) microinjection of spirochetes directly into the tick gut and (iv) capillary or membrane feeding of ticks with a solution or blood containing Borrelia species. organisms. To reduce the use of live animals and for standardization of the conditions of experiments, the three latter procedures are recommended. The present study aimed to investigate the effectiveness of an immersion procedure to generate $B$. burgdorferi-infected ticks and determine whether Ixodes scapularis and I. ricinus ticks were infective to dogs. Pathogen free, unfed larvae (I. scapularis and I. ricinus) and nymphs (I. scapularis) were immersed in BSK-H medium containing approximately $10^{7}$ B. burgdorferi sensu stricto (strain B31) organisms per mL. Immersed ticks were then fed to repletion on rabbits and held under optimum environmental conditions $\left(22^{\circ} \mathrm{C}\right.$ and $80 \pm 10 \%$ relative humidity) for moulting. The infection rate in ticks was determined after moulting by qPCR, while their potential infectivity was evaluated on dogs. It was found that immersed larvae and nymphs acquired spirochetes. The spirochetes were detected by qPCR in $18.7 \%$ and $37.5 \%$ of adult $I$. ricinus and I. scapularis, respectively. For nymphs, B. burgdorferi-specific DNA was detected in each of three pools of 20 I. scapularis. Nevertheless, all infested dogs remained seronegative during the three months after infestation and no clinical signs of borreliosis were detected.
\end{abstract}

Keywords: Borrelia burgdorferi; Ixodes scapularis; Ixodes ricinus; Immersion Technique; Transmission, Dogs

\section{Abbreviations}

ss: Sensu Stricto; LB: Lyme Borreliosis; PBS: Phosphate-Buffered Saline; qPCR: Quantitative Polymerase Chain Reaction; Ct: Cycle Threshold

\section{Introduction}

Lyme borreliosis (LB) is caused by spirochetes belonging to the complex of Borrelia burgdorferi sensu lato. These agents are trans- 
mitted to humans, dogs and other animals by infected Ixodes species ticks. In Europe, approximately 40 mammal and bird species are reservoirs/hosts of $B$. burgdorferi [1]. Small rodents, especially Apodemus sp. and Clethrionomys sp., serve as important hosts for the immature stages of $I$. ricinus and as sources of infection with $B$. burgdorferi [2]. In the eastern USA, the white-footed mouse Peromyscus leucopus is considered the primary host and a source of infection for I. scapularis. Other small mammals such as shrews and chipmunks are also an important reservoirs [3,4]. Although dogs are not reservoirs, they can be infected by these pathogens. Their susceptibility to the infection with Borrelia organisms make dogs relevant epidemiological sentinels [5]. In endemic areas LB represents a potential health threat to dogs. LB in dogs is characterized by non-specific clinical signs such as fever, anorexia, lethargy and lymphadenopathy [6]. More severe signs, such as arthritis with lameness, neurologic disorders, and glomerulonephritis can also be observed in dogs $[7,8]$.

Laboratory generation of $B$. burgdorferi-infected ticks used in tick-host-pathogen interaction studies of LB usually requires an animal model. Mice are used most often, because they are natural biological reservoirs. Decreased use of animals in scientific research is desirable for animal well-being. Alternative in vitro procedures would allow more effective standardization of techniques and procedures. To date, I. scapularis and I. ricinus ticks infected with $B$. burgdorferi were generated using in vitro methods such as immersion of ticks in solution containing spirochetes [9,10], microinjection of spirochetes directly into the tick gut [11,12], capillary feeding of ticks with solution containing B. burgdorferi $[13,14]$ and feeding on artificial membranes [11,12].

In this study, we investigated the effectiveness of an immersion procedure to generate I. scapularis and I. ricinus ticks infected with B. burgdorferi. We also evaluated the capability of ticks that were infected by this technique to transmit spirochetes to dogs.

\section{Materials and Methods}

Ethics approval

The protocol was reviewed and approved by the study site Institutional Animal Care and Use Committee (IACUC) prior to implementation (approval no. MDM-001-19).

\section{Ticks and bacteria}

Nymphs and larvae of I. scapularis and I. ricinus were obtained from a laboratory colony initiated in 2012 using adult specimens collected in the field from Georgia (USA) and Utrecht (the Netherlands). Pathogen-free tick are maintained by routine passage on rabbits for larvae and nymphs, and on sheep for adult ticks. Ticks are incubated at $12^{\circ} \mathrm{C}$ for storage or $22^{\circ} \mathrm{C}$ for development and at $80 \pm 10 \%$ relative humidity. To increase their willingness to feed, ticks were transferred under environmental conditions one week before challenge. A low passage (P3) strain of B. burgdorferi ss (strain B31, ATCC 35210) was used to infect ticks. Borrelia cultures were maintained in complete $\mathrm{BSK}-\mathrm{H}$ media at $34^{\circ} \mathrm{C}$ as described previously [15].

\section{Immersion of ticks in suspensions of Spirochetes}

The immersion protocol was from Policastro and Schwan [9]. About 800 starved larvae or nymphs of each Ixodes species (I. scapularis or I. ricinus) were introduced into 3-mL tubes containing 2 $\mathrm{mL}$ of BSK-H medium with $10^{7}$ Borrelia cells per mL. Spirochete cell concentrations were determined by counting spirochetes in defined suspension volumes using a dark-field microscope. Tubes containing ticks were incubated for 2 hours at $34^{\circ} \mathrm{C}$ and vortexed gently every 15 minutes. After immersion, ticks were rinsed twice with PBS, dried on filter paper and then kept in an incubator until they were fed on specific pathogen-free rabbits. Detached and fully engorged ticks were incubated until they molted to the next stage.

Detection of $B$. burgdorferi ss DNA by polymerase chain reaction

The infection status of I. scapularis and I. ricinus immersed ticks was determined after moulting by real-time polymerase chain reaction (qPCR) as described previously [16]. DNA was extracted from whole ticks using a NucleoSpin tissue kit according to the manufacturer's recommendations (Macherey-Nagel, Hoerdt, France). Adults were tested individually while nymphs were tested by pooling 20 specimens. DNA-free water and DNA from uninfected ticks were used as negative controls. Borrelia burgdorferi DNA were used as positive controls. The samples were considered positive when the threshold cycles $(\mathrm{Ct})$ were inferior to 38.5 .

Ability of infected ticks to transmit infections to dogs

Six adult male beagle dogs (aged 2 - 4 years, weighing 10.4 - 13.3 $\mathrm{kg}$ ) were used: two dogs were infested with nymphal I. scapularis ( $\mathrm{N}=100$ per dog), two with 60 (40 females and 20 males) adult I. scapularis per dog and two others with 60 (40 females and 20 males) adult I. ricinus per dog. Ticks were introduced inside patches previously glued to a dorsolateral area near the ribcage of each dog (Figure 1). Dogs were observed daily for the development of 
Assessment of an Immersion Technique for Generating of Borrelia burgdorferi-Infected and Infectious Ixodes scapularis and Ixodes ricinus Ticks

161

any signs of pain, lameness, lethargy, or anorexia. Blood was collected from each dog on days $24,38,52,66,80$ and 94 post infestation and tested for B. burgdorferi-specific antibodies (SNAP ${ }^{\circledR} 4 \mathrm{DX}^{\circledR}$ Plus, IDEXX Laboratories, Westbrook Maine, USA). The sensitivity and specificity of this assay is $98.8 \%$ and $100 \%$, respectively [17].

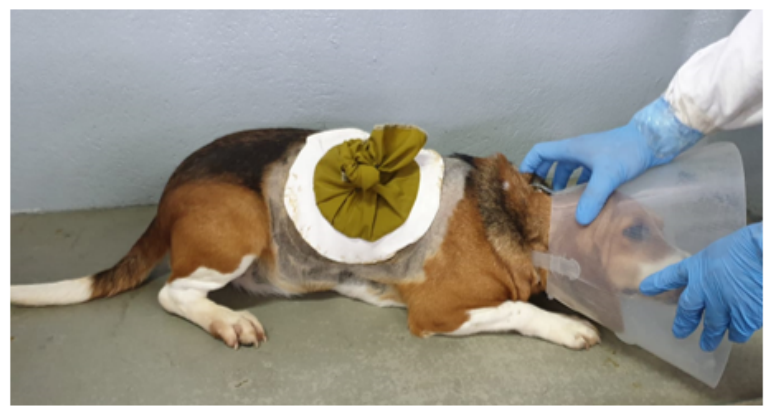

(a)

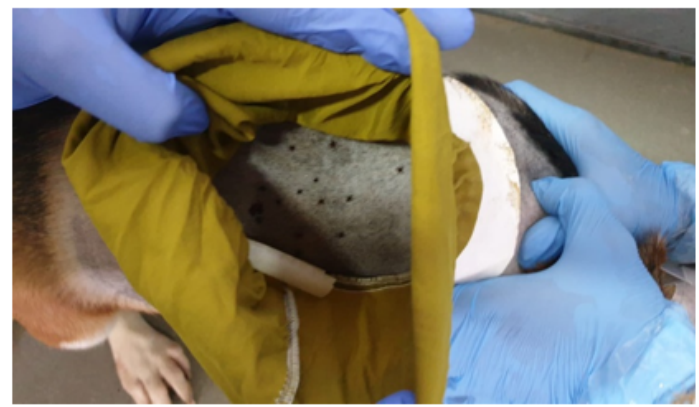

(b)

Figure 1: Tick infestation procedure: (a) Patches glued to the dorsolateral region; (b) Infestation with B. burgdorferi-infected I. scapularis/or I. ricinus ticks.

\section{Results}

Borrelia burgdorferi-specific DNA was detected successfully in the molted ticks. The infection rate was $18.7 \%$ and $37.5 \%$ for adult I. ricinus and I. scapularis ticks, respectively. In the case of I. scapularis nymphs, Borrelia burgdorferi specific DNA was detected in all three pools of nymphs (Table 1). Of the positive samples, only one pool of I. scapularis ticks was moderately loaded with $B$. burgdorferi (Ct value $=26.7$ corresponding to $5 \times 10^{3}$ spirochetes), while the others were weakly loaded (Ct values ranging between 34.4 and 38.2 ( $\leq 50$ spirochetes).

\begin{tabular}{|c|c|c|c|}
\hline Tick species & \multicolumn{2}{|c|}{ Ixodes scapularis } & Ixodes ricinus \\
\hline Stage & Nymphs* & Adults & Adults \\
\hline No. tested ticks & 3 & 32 & 32 \\
\hline No. infected ticks & 3 & 12 & 6 \\
\hline Infection rate (\%) & $/$ & 37.5 & 18.7 \\
\hline
\end{tabular}

Table 1: Borrelia burgdorferi infection of nymphal and adult I. scapularis and I. ricinus ticks by immersion technique (qPCR results).

*: The nymphal stage was tested in pools; each pool contained 20 specimens. So, a total of 60 nymphs were tested.

Adults and nymphal Borrelia-exposed ticks attached quickly and fed well on the dogs. The in-situ count performed $48 \mathrm{~h}$ after tick infestation, demonstrated an attachment rate of $80.8 \%(97 / 120)$ and $64.1 \%(86 / 120)$ for adult I. scapularis and I. ricinus, respectively. For nymphal $I$. scapularis ticks, the attachment was lower at $44.0 \%(88 / 200)$.

None of the infested dogs seroconverted throughout the threemonth follow-up observation period, nor were clinical signs such as lameness, anorexia, weakness, or elevated body temperature detected in these dogs.

\section{Discussion and Conclusion}

The development of laboratory methods for infecting ticks with different pathogens of veterinary importance is a key step for further research. The establishment of in vitro models would aid in advancing our knowledge of tick-pathogen interactions and would reduce animal use. We chose the immersion technique for infecting ticks with B. burgdorferi for several reasons. Firstly, this technique was validated in previous laboratory studies using I. scapularis and I. ricinus ticks $[9,10,18]$; secondly, this procedure allows the production of large numbers of infected ticks without the need for infecting host animals; and thirdly, it does not require any special equipment compared to the other more sophisticated procedures (e.g. microinjection).

The infection rate obtained here for I. ricinus was lower (18\%) compared to those obtained for I. scapularis (37\%) or to the infection rate of $65 \%$ reported by Fiserova., et al. [10] using PCR. Even though the detection techniques were different, the infection rate $(37.5 \%)$ in our study for I. scapularis, was similar to those reported (45\% - 65\%) by Policastro and Schwan [9], using an immunofluorescence assay. 
The vector competence of I. scapularis and I. ricinus for B. burgdorferi, strain B31 was investigated previously [19,20]. The susceptibility of dogs to $B$. burgdorferi ss infection using naturally infected ticks was also reported in numerous studies [7,21,22]. In the latter studies, seroconversion was observed from day 10 onward, with maximum titers occurring within 50 to 90 days after infestation. In another published study, the successful transmission of $B$. burgdorferi infection to dogs was documented after ticks were artificially infected with I. scapularis by capillary feeding [23]. The authors reported that $100 \%(5 / 5)$ and $20 \%(1 / 5)$ of dogs seroconverted when they were infested with adults and nymphal I. scapularis, respectively. Our decision to use dogs to demonstrate transmission by molted ticks was based on these study results.

Ticks in this study failed to induce infections with $B$. burgdorferi ss in dogs, even though we used the same described immersion technique and infection in immature/adult I. scapularis and I. ricinus was demonstrated. This could be explained by a low spirochete load in immersed ticks after moulting ( $\mathrm{Ct}>26$ ). It has been reported that dogs exposed to naturally infected, field-collected I. scapularis nymphs had lower rates of seroconversion than those infected using adult ticks [21]. This may reflect the smaller number of bacteria transmitted by nymphs during feeding [23]. Interestingly, I. ricinus nymphs derived from immersed larvae did not induce seroconversion when they fed on $\mathrm{C} 3 \mathrm{H}$ mice [18]. In the present study we did not confirm the viability of $B$. burgdorferi ss bacteria in ticks after their moult to the nymphal/adult stage. Thus, failure to induce infections in dogs might be due to the low bacterial load or to the absence of viable spirochetes in the molted ticks.

Our findings demonstrate that immature/adult I. scapularis and $I$. ricinus ticks can acquire B. burgdorferi while immersed in a suspension containing spirochetes. Although spirochetes may have survived the moult to subsequent nymphal or adult life stages, molted ticks were unable to transmit the infection to laboratory beagle dogs.

\section{Acknowledgments}

The authors extend their appreciation to the personnel at the study site for their assistance in the conduct of this study.

\section{Conflicts of Interest Statement}

All the authors declare no conflicts of interest related to this article.

\section{Authors' Contributions}

DT and MV conceived the study and designed the experiments. DT, AE and NL performed the experiments. DT drafted the manuscript. All authors read and approved the final version of the manuscript.

\section{Bibliography}

1. Gern L., et al. "European reservoir hosts of Borrelia burgdorferi sensu lato". Zentralblatt Fur Bakteriologie: International Journal of Medical Microbiology 287 (1998): 196-204.

2. Lane RS., et al. "Lyme borreliosis: relation of its causative agent to its vectors and hosts in North America and Europe". Annual Review of Entomology 36 (1991): 587-609.

3. Brisson D., et al. "Conspicuous impacts of inconspicuous hosts on the Lyme disease epidemic". Proceedings Biological Sciences 275 (2008): 227-235.

4. Marchant A., et al. "Infection of Ixodes ricinus by Borrelia burgdorferi sensu lato in peri-urban forests of France". PLOS ONE 12 (2017): e0183543.

5. Herrin BH., et al. "Canine and human infection with Borrelia burgdorferi in the New York City metropolitan area". Parasites and Vectors 11 (2018): 187.

6. Krupka I and Straubinger RK. "Lyme Borreliosis in Dogs and Cats: Background, Diagnosis, Treatment and Prevention of Infections with Borrelia burgdorferi sensu stricto". Veterinary Clinics of North America - Small Animal Practice 40 (2010): 1103-1119.

7. Straubinger RK., et al. "Clinical manifestations, pathogenesis, and effect of antibiotic treatment on Lyme borreliosis in dogs". Wiener Klinische Wochenschrift 110 (1998): 874-881.

8. Dambach DM., et al. "Morphologic, Immunohistochemical, and Ultrastructural Characterization of a Distinctive Renal Lesion in Dogs Putatively Associated with Borrelia burgdorferi Infection: 49 Cases (1987-1992)". Veterinary Pathology 34 (1997): $85-96$.

9. Policastro PF and Schwan TG. "Experimental infection of Ixodes scapularis larvae (Acari: Ixodidae) by immersion in low passage cultures of Borrelia burgdorferi". Journal of Medical Entomology 40 (2003): 364-370. 
10. Fiserova L., et al. "Two ways of experimental infection of $I x o$ des ricinus ticks (Acari: Ixodidae) with spirochetes of Borrelia burgdorferi sensu lato complex". Folia Parasitologica 55 (2008): 150-154.

11. Kariu T., et al. "Methods for Rapid Transfer and Localization of Lyme Disease Pathogens Within the Tick Gut". Journal of Visualized Experiments (2011).

12. Smith AA., et al. "Artificial Infection of Ticks with Borrelia burgdorferi Using a Microinjection Method and Their Detection In Vivo Using Quantitative PCR Targeting flaB RNA". Methods In Molecular Biology 1690 (2018): 105-114.

13. Broadwater AH., et al. "Glass capillary tube feeding: a method for infecting nymphal Ixodes scapularis (Acari: Ixodidae) with the lyme disease spirochete Borrelia burgdorferi". Journal of Medical Entomology 39 (2002): 285-292.

14. Oliver JD., et al. "Infection of Immature Ixodes scapularis (Acari: Ixodidae) by Membrane Feeding". Journal of Medical Entomology 53 (2016): 409-415.

15. Barbour AG. "Isolation and cultivation of Lyme disease spirochetes". The Yale Journal of Biology and Medicine 57 (1984): 521-525.

16. Courtney JW., et al. "Multiplex real-time PCR for detection of Anaplasma phagocytophilum and Borrelia burgdorferi". Journal of Clinical Microbiology 42 (2004): 3164-3168.

17. Chandrashekar R., et al. "Performance of a commercially available in-clinic ELISA for the detection of antibodies against Anaplasma phagocytophilum, Ehrlichia canis, and Borrelia burgdorferi and Dirofilaria immitis antigen in dogs". American Journal of Veterinary Research 71 (2010): 1443-1450.

18. Horká H., et al. "Efficiency of experimental infection of Ixodes ricinus ticks with Borrelia burgdorferi spirochetes". International Journal of Medical Microbiology 298 (2008): 177-179.

19. Dolan MC., et al. "Vector Competence of Ixodes scapularis and Ixodes ricinus (Acari: Ixodidae) for Three Genospecies of Borrelia burgdorferi". Journal of Medical Entomology 35 (1998): 465-470.
20. Sertour N., et al. "Infection Kinetics and Tropism of Borrelia burgdorferi sensu lato in Mouse After Natural (via Ticks) or Artificial (Needle) Infection Depends on the Bacterial Strain". Frontiers in Microbiology 9 (2018): 1722.

21. Appel MJG., et al. "Experimental lyme disease in dogs produces arthritis and persistent infection". Journal of Infectious Diseases (1993).

22. Greene RT., et al. "Clinical and serologic evaluations of induced Borrelia burgdorferi infection in dogs". American Journal of Veterinary Research 49 (1988): 752-757.

23. Burkot TR., et al. "Quantitation of the Borrelia burgdorferi outer surface protein A in Ixodes scapularis: fluctuations during the tick life cycle, doubling times, and loss while feeding". The Journal of Infectious Diseases 170 (1994): 883-889.

\section{Assets from publication with us}

- Prompt Acknowledgement after receiving the article

- Thorough Double blinded peer review

- Rapid Publication

- Issue of Publication Certificate

- High visibility of your Published work

Website: www.actascientific.com/

Submit Article: www.actascientific.com/submission.php

Email us: editor@actascientific.com

Contact us: +919182824667 